\title{
Violência Autoinfligida na Ótica da Equipe de um Programa de Transtornos Alimentares
}

\author{
Self-Inflicted Violence from the Perspective of an Eating Disorders Program Team
}

Violencia Autoinfligida desde la Perspectiva de un Equipo del Programa de Trastornos de la Alimentación

Cléa Adas Saliba GARBIN

Departamento de Odontologia Preventiva e Restauradora, Área de Saúde Coletiva, Faculdade de Odontologia de Araçatuba (FOA) Universidade Estadual Paulista (UNESP) 16015-050 Araçatuba - SP, Brasil https://orcid.org/0000-0001-5069-8812 Ronald Jefferson MARTINS

Departamento de Odontologia Preventiva e Restauradora, Área de Saúde Coletiva, Faculdade de Odontologia de Araçatuba (FOA), Universidade Estadual Paulista (UNESP) 16015-050 Araçatuba - SP, Brasil https://orcid.org/0000-0001-8908-3524

Naiana de Melo BELILA

Departamento de Odontologia Preventiva e Restauradora, Área de Saúde Coletiva, Faculdade de Odontologia de Araçatuba (FOA), Universidade Estadual Paulista (UNESP) 16015-050 Araçatuba - SP, Brasil https://orcid.org/0000-0002-1099-9116

Tânia Adas SALIBA

Departamento de Odontologia Preventiva e Restauradora, Área de Saúde Coletiva, Faculdade de Odontologia de Araçatuba (FOA), Universidade Estadual Paulista (UNESP) 16015-050 Araçatuba - SP, Brasil https://orcid.org/0000-0003-1327-2913 Artênio José Ísper GARBIN

Departamento de Odontologia Preventiva e Restauradora, Área de Saúde Coletiva, Faculdade de Odontologia de Araçatuba (FOA), Universidade Estadual Paulista (UNESP) 16015-050 Araçatuba - SP, Brasil https://orcid.org/0000-0002-7017-8942

\section{Resumo}

Introdução: A autopunição é aquela que ocorre quando uma pessoa pratica uma ação consciente de autodestruição (violência autoinfligida) e pode ser subdividida em comportamento suicida e em autoagressão. Objetivo: Verificou-se a violência autoinfligida na fala da equipe de saúde de pacientes com anorexia e bulimia nervosa do Programa de Transtornos Alimentares do Ambulatório de Saúde Mental de uma Instituição Pública de Ensino. Materiais e métodos: Trata-se de uma pesquisa qualitativa realizada com médicos, psicólogos e cirurgião-dentista $(n=9)$, pertencentes à equipe de saúde do programa. $O$ grupo focal teve a participação de um moderador, observador e a equipe de saúde, empregando-se um roteiro com questões norteadoras. Os discursos foram gravados, transcritos e os conteúdos textuais processados no software IRAMUTEQ e analisados pela técnica da Nuvem de Palavras e Análise de Similitude. Resultados: Foram observadas 2.541 ocorrências de palavras, sendo 264 formas distintas. As palavras que obtiveram frequência relativa no corpus textual na primeira técnica foram: suicídio, não, muito, mutilar e burlar; e na segunda, o leque semântico central de palavras foi: muito, não, suicídio, mutilar e burlar. Conclusão: Conclui-se que a violência autoinfligida mostra-se muito presente entre mulheres com anorexia e bulimia nervosa.

Descritores: Transtornos da Alimentação e da Ingestão de Alimentos; Violência; Anorexia; Bulimia Nervosa.

\section{Abstract}

Introduction: Self-punishment is that which occurs when a person commits a conscious action of self-destruction (self-inflicted violence) and can be subdivided into suicidal behavior and self-harm. Objective: Self-inflicted violence was verified in the speech of the healthcare team of patients with anorexia and bulimia nervosa in the Eating Disorders Program of the Mental Health Clinic of a Public Education Institution. Materials and methods: This is a qualitative research carried out with physicians, psychologists and dentists $(n=9)$, belonging to the program's health team. The focus group had the participation of a moderator, observer and the health team, using a script with guiding questions. The speeches were recorded, transcribed and the textual contents processed in the IRAMUTEQ software and analyzed using the Word Cloud and Similarity Analysis technique. Results: There were 2,541 occurrences of words, with 264 distinct forms. The words that obtained relative frequency in the textual corpus in the first technique were: suicide, no, very much, mutilating and cheating; and in the second, the central semantic range of words was: much, no, suicide, mutilate and cheat. Conclusion: It is concluded that self-inflicted violence is very present among women with anorexia and bulimia nervosa.

Descriptors: Feeding and Eating Disorders; Violence; Anorexia; Bulimia Nervosa.

\section{Resumen}

Introducción: El autocastigo es aquel que ocurre cuando una persona practica una acción consciente de autodestrucción (violencia autoinfligida) y puede subdividirse en conducta suicida y autolesión. Objetivo: Se verificó la violencia autoinfligida en el discurso del equipo de salud de pacientes con anorexia y bulimia nerviosa en el Programa de Trastornos de la Alimentación de la Clínica de Salud Mental de una Institución de Educación Pública. Materiales y métodos: Se trata de una investigación cualitativa realizada con médicos, psicólogos y odontólogos $(n=9)$, pertenecientes al equipo de salud del programa. El grupo focal contó con la participación de un moderador, un observador y el equipo de salud, utilizando un guión con preguntas orientadoras. Los discursos fueron grabados, transcritos y el contenido textual procesado en el software IRAMUTEQ y analizado mediante la técnica Word Cloud y Similarity Analysis. Resultados: Hubo 2.541 ocurrencias de palabras, con 264 formas distintas. Las palabras que obtuvieron relativa frecuencia en el corpus textual en la primera técnica fueron: suicidio, no, mucho, mutilar y engañar; y en el segundo, el rango semántico central de palabras fue: mucho, no, suicidio, mutilación y trampa. Conclusión: Se concluye que la violencia autoinfligida está muy presente entre las mujeres con anorexia y bulimia nerviosa.

Descriptores: Trastornos de Alimentación y de la Ingestión de Alimentos; Violencia; Anorexia; Bulimia Nerviosa.

INTRODUÇÃO

Os transtornos alimentares têm sido amplamente estudados pelos especialistas da saúde, tanto nas áreas da medicina e biologia, como também na sociologia e psicologia'. Revelam-se pela abundância ou déficit na ingestão alimentar, devido uma distorção em nível cognitivo ou perceptivo por parte dos indivíduos que os apresentam. Em qualquer um dos casos deixa de ser realizada uma alimentação saudável e equilibrada ${ }^{2}$. 
De acordo com os manuais de classificação diagnóstica internacional, da Associação Psiquiátrica Americana (APA) ${ }^{3}$ e da Classificação Estatística Internacional de Doenças e Problemas Relacionados à Saúde $(\mathrm{CID}-10)^{4}$ são considerados atualmente como transtornos alimentares: a anorexia, a bulimia nervosa, o transtorno de compulsão alimentar periódica, a perda de apetite, a hiperfagia ou vômitos de origem psicogênica.

Dentre os principais temos a anorexia e a bulimia nervosa. A primeira é definida como um distúrbio de ordem comportamental caracterizado pela inanição deliberada e autoimposta, seguida da busca constante pela magreza e por um medo mórbido de engordar, levando a graves níveis de emagrecimento. Já a bulimia, caracteriza-se por episódios recorrentes e incontroláveis de consumo de grandes quantidades de alimento em curto período de tempo, seguidos de comportamentos compensatórios inadequados, como vômitos autoinduzidos, uso de laxantes e diuréticos, exercícios vigorosos, jejum e dieta restritiva ${ }^{5}$.

As condutas dos pacientes em relação a esses transtornos alimentares são bastante nefastas, pois estes recorrem a métodos agressivos em busca do peso que consideram como ideal, podendo originar graves problemas de saúde ${ }^{2}$. Essas "técnicas" são práticas de autopunições diante da fome ou da compulsão, que podem ser desde o autoflagelo até a busca pelo emagrecimento por meio da sangria ${ }^{1}$.

A autopunição é aquela que ocorre quando uma pessoa pratica uma ação consciente de autodestruição, conhecida como violência autoinfligida e pode ser subdividida em comportamento suicida e em autoagressão. $O$ primeiro inclui pensamentos suicidas, tentativas e suicídios propriamente ditos, enquanto que a autoagressão engloba atos como a automutilação ${ }^{6}$.

A escolha do meio utilizado nas violências autoinfligidas abrange aspectos psicossociais, de gênero, aceitabilidade sociocultural, além da disponibilidade e acesso aos materiais para o autoflagelo ${ }^{6}$.

A prática autolesiva tem despertado cada vez mais o interesse dos pesquisadores, professores e profissionais da saúde, pois muitas vezes um ato inconsciente de escarificar a pele resulta em um suicídio não intencional. Devido ao grande número de ocorrência dos casos de automutilação, esta ação foi definida como um problema de saúde pública, por muitas vezes 0 ato estar relacionado a distúrbios alimentares ${ }^{7}$.

Ressalta-se que o desfecho não fatal configura-se, geralmente, como uma urgência e emergência médica, o que exige intervenções precisas, eficazes e dotadas de efetividade a curto e médio prazo. Dessa forma, os programas de atendimento aos transtornos alimentares apresentam-se como uma importante fonte de informação, essencial para o planejamento de serviços e ações preventivas $^{6,8}$.

Baseado neste contexto, o presente trabalho teve por objetivo analisar a violência autoinfligida por mulheres com anorexia e bulimia nervosa na fala da equipe de saúde multidisciplinar de um Programa de Transtornos Alimentares.

\section{MATERIAL E MÉTODO}

Trata-se de uma pesquisa qualitativa, de caráter transversal e com o emprego da técnica de grupo focal. A amostra foi composta por 9 integrantes da equipe de saúde; entre eles, médicos, psicólogos e cirurgião dentista; pertencentes a um Programa de Transtornos Alimentares do Ambulatório de Saúde Mental de uma Instituição Pública de Ensino Superior, no ano de 2018.

O grupo focal foi realizado com a participação de um moderador e observador, e a equipe de saúde. Para conduzir a entrevista, o moderador utilizou um roteiro de tópicos, elaborado para nortear a conversa sobre a seguinte temática: "A violência autoinfligida por mulheres com anorexia e bulimia nervosa".

Para a realização da atividade, foram tomadas algumas medidas, como: preparo da sala, com acomodações e condições adequadas para os participantes; sinalização no lado de fora da sala ("Não perturbe, estamos em reunião"), a fim de que não houvesse interrupção das atividades do grupo; verificação do material a ser utilizado para gravação de áudio; e a recepção adequada dos integrantes do grupo.

No início da reunião, foram tomadas algumas medidas de acordo com as normas da resolução 466/12, como: explicação para os participantes sobre os objetivos da pesquisa e da técnica utilizada; solicitação para o uso do gravador de áudio; organização das falas e sua importância; e o anonimato.

Os entrevistados receberam um número ao início da atividade, para que dessa forma não fossem identificados durante a conversa e para que o direcionamento das perguntas do roteiro ocorresse de forma mais dinâmica. $O$ grupo focal teve duração de 30 minutos, pois foi o tempo permitido pela coordenação do ambulatório para a execução da atividade.

A técnica de entrevista em grupo focal 
baseia-se no diálogo integrativo entre os participantes e pesquisador, tendo desta forma o objetivo de apreender o entendimento de diferentes representações sociais, referente a uma prática, fato, produto ou serviço, a partir da discussão focada em tópicos específicos e diretos ${ }^{9}$.

Os discursos gravados foram transcritos e os conteúdos textuais processados no software IRAMUTEQ (Interface de $\mathrm{R}$ pour lês Analyses Multidimensionnelles de Textes et de Questionnaires), que consiste em um programa livre que se ancora no software $R$, e que permite processamento e análises estatísticas de textos produzidos. Foi desenvolvido por Ratinaud ${ }^{10}$ na língua francesa, mas atualmente possui tutoriais completos em outras línguas, inclusive 0 português.

Os dados foram observados por meio de análise descritiva e pelas técnicas da Nuvem de Palavras e Análise de Similitude. Foram removidos da listagem de palavras de conteúdo do corpus textual os pronomes pessoais, de tratamento e possessivos que apareceram em grande frequência, para que desta forma os resultados fossem apresentados de forma mais clara.

A pesquisa foi aprovada pelo Comitê de Ética em Pesquisa, com parecer CAAE 80497417.1.0000.5420.

\section{RESULTADOS}

A análise do corpus textual "A violência autoinfligida por mulheres com anorexia e bulimia nervosa" proveniente do grupo focal, proporcionou uma ocorrência de 2.541 palavras de conteúdo; sendo destas, 264 formas distintas.

A partir de uma análise descritiva e interpretativa dos dados, pode-se verificar a existência de grande ocorrência da violência autoinfligida nos pacientes com anorexia e bulimia nervosa. Fato esse constatado de forma expressiva por meio do conteúdo do grupo focal. Algumas falas da equipe de saúde demonstraram tal interpretação:

[...] Ela pegou o marido com outra, e depois disso todos os problemas começaram, tinha um quadro grave de depressão. Começou com os vômitos e agora foi diagnosticada com bulimia. Ela tentou o suicídio tomando cartelas de medicações e agora disse que ficou parada por uns vinte minutos em cima da ponte, pensando em se jogar (Participante 1).
[...] As pacientes se mutilam, isso ocorre com uma frequência muito grande. Eu tive uma paciente que ia na farmácia e comprava seringas para tirar o próprio sangue, porque ela achava que isso iria fazer ela emagrecer. Não se importava com essa mutilação. $\mathrm{Na}$ verdade elas não entendem dessa forma. Ela tirava várias seringas de sangue por dia. Outra paciente também se mutilava, ela fazia cortes no próprio corpo, onde sangrava bastante, na parte de dentro da coxa, com lâminas de barbear. Esse método é realizado por várias pacientes, mas é muito difícil de descobrir pelo local ficar escondido (Participante 3).

Outras falas mostram a resistência das pacientes em aceitarem o tratamento proposto e métodos utilizados para burlar as ingestões de alimentos.

[...] Elas escondem a comida dentro da roupa, dentro do guarda roupas, elas burlam o atendimento quando são forçadas a comer. Elas conseguem fazer com que você se distraia, manipulando e burlando uma situação, pra conseguir sair disso e jogar a comida fora. Muitas vezes nos hospitais precisamos deixar trancada a porta do banheiro, pra elas não jogarem a comida no vaso e dar descarga. Elas sempre encontram um jeito de burlar o sistema que as ajuda (Participante 6).

[...] $\mathrm{Na}$ internação ela escondia a comida, jogava fora no vaso do banheiro, esconde até no quarto dos vizinhos. Ela faz um tipo de sedução para você acreditar no que ela diz, para achar que ela está bem e se livrar do tratamento. Ela mente muito! (Participante 7)

$\mathrm{Na}$ análise pela Nuvem de Palavras, ocorre um agrupamento e organização gráfica em função de sua frequência, possibilitando assim a rápida identificação das palavras-chave do corpus textual e análise lexical simples ${ }^{11}$. Foi observado que as palavras que possuíram frequência relativa foram: "suicídio, não, muito, mutilar e burlar", constatando, 32, 30, 26, 20 e 20 vezes respectivamente, na transcrição do corpus textual. (Figura 1) 
DISCUSSÃO

\section{emagrecer mutilação \\ banheiro possuir comermutilar $\beth_{\tilde{E}_{\text {sofrer }}}^{\tilde{\omega}_{\text {s. }}}$ quererburlar $\varepsilon_{\text {sonda }}^{\text {mae }}$ ouar metiformina morrer comida conseguircorpo esconder}

Figura 1. Nuvem de palavras do contexto "A violência autoinfligida por mulheres com anorexia e bulimia nervosa", Marília, 2018.

Foi realizada também a análise de Similitudes, que se ancora na teoria dos grafos, possibilitando a identificação da ocorrência entre as palavras e seu resultado nas indicações da conexidade entre as mesmas, auxiliando na identificação da estrutura da representação ${ }^{12}$.

$\mathrm{Na}$ figura 2, podemos observar que ocorreu um leque semântico das palavras mais frequentes: "muito, não, suicídio, mutilar e burlar" e suas conexidades.

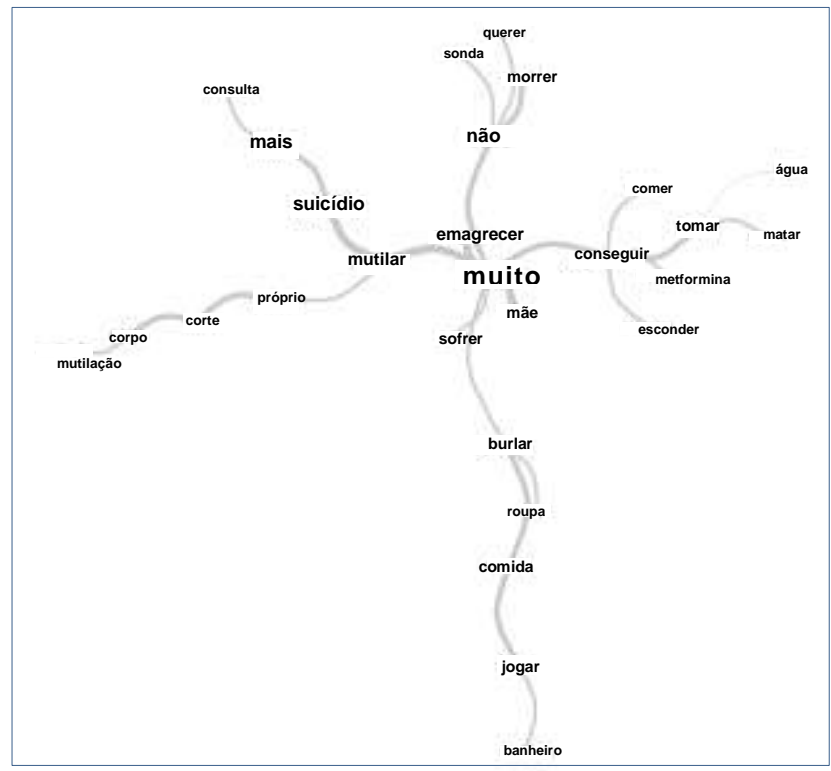

Figura 2. Análise de Similitudes entre as palavras do contexto "A violência autoinfligida por mulheres com anorexia e bulimia nervosa", Marília, 2018.

Desta forma, os discursos mostram um alto índice de automutilação e tentativas de suicídio entre as mulheres diagnosticadas com anorexia e bulimia nervosa, bem como métodos para burlarem o tratamento.
Os transtornos alimentares são síndromes comportamentais cujos critérios diagnósticos têm sido amplamente estudados. Apresentam-se descritos como transtornos e não como doenças por ainda não ser bem conhecida sua etiopatogenia. Sabe-se da sua multifatoriedade, podendo ser determinada por fatores genéticos, psicológicos e socioculturais, caracterizando-se, portanto, como transtornos biopsicossociais $^{13}$.

$\mathrm{Na}$ atualidade, constituem verdadeira epidemia, onde um número alarmante de pessoas sofre com esses tipos de distúrbios, na proporção de 10:1 para o gênero feminino e uma faixa etária média de 25 anos ${ }^{14,15}$.

Os dois principais transtornos alimentares encontrados na sociedade atualmente são a anorexia e a bulimia nervosa, que podem levar os indivíduos, nos casos mais graves, a atitudes violentas contra si mesmas ${ }^{14}$.

Este comportamento está associado a mecanismos de enfrentamento ou estratégias para a regulação de suas emoções, neste sentido a automutilação deliberada pode ser utilizada para alívio de emoções muito fortes, diminuição da tensão e até mesmo a autopunição ${ }^{16}$.

De acordo com a Sociedade Internacional de Estudo da Violência Autodirigida $(\mathrm{DSM}-5)^{3}$, são definidos como fatores de risco que podem desencadear o comportamento autolesivo, as perturbações psicopatológicas e história de suicídio na família; negligência e maus tratos na infância; expectativas demasiadamente elevadas ou baixas dos pais em relação aos filhos; excesso de autoridade; rigidez familiar; divórcios e/ou separações; dificuldades escolares; conflitos interpessoais e problemas de relacionamento; separação de amigos/colegas/companheiro; e a morte de pessoas próximas do seu convívio. Existem também fatores tidos como protetores desses comportamentos; entre eles, as boas relações familiares e competências sociais, existência de um bom suporte social, adoção de estilo de vida saudáveis, identificação efetiva com valores culturais; além de boas relações interpessoais e grupais ${ }^{3,17,18}$.

A análise das falas da equipe de saúde possibilitou averiguar vários episódios de violência autoinfligida cometida pelas mulheres com anorexia e bulimia nervosa, por meio de discursos e ocorrência das palavras "suicídio, mutilar e muito". Estudo realizado por Farah \& Mate ${ }^{1}$, revelou tipos de práticas de emagrecimento, dentre elas, a Técnica de autopunição, que visava à manutenção do peso 
e das formas corporais, por meio de práticas autolesivas diante da fome ou da compulsão, que iam desde 0 autoflagelo até $a$ automutilação, a partir do relato de jovens anoréxicas e bulímicas no espaço virtual.

O principal método relatado para tal ação nos resultados do presente estudo foi o de cortes com lâminas de aço na região interna das coxas, o que corrobora os achados de Venosa $^{19}$, onde as mulheres se cortavam na coxa com lâminas, no intuito de remover sua própria gordura e perder sangue para emagrecer, denominando de autolipoaspiração, e outras para a autopunição. No entanto, o estudo de Pinaud e colaboradores ${ }^{20}$, relatou que - local corporal mais acometido por automutilação é a parte interna do antebraço, vindo às coxas em segundo lugar e que as mulheres pesquisadas utilizavam sua própria pele como superfície visível de seu sofrimento interno.

Presume-se que tal local seja escolhido, por ser de difícil visualização pela equipe de saúde, familiares e pessoas mais próximas, podendo assim manter as feridas e cicatrizes escondidas por se tratar de um local que sempre estará coberto.

O presente trabalho foi realizado somente com mulheres devido ser a quase totalidade dos casos no ambulatório pesquisado, o que vai ao encontro dos relatos de outros autores que mostram que a violência autoinfligida é duas vezes mais presente entre as mulheres, apesar do crescente número de homens com esses transtornos na atualidade. Relatam também que a prática costuma ter início após a puberdade, estando principalmente entre os bulímicos ${ }^{20-22}$.

Vários fatores

ocorrendo concomitantemente levam os indivíduos a desencadearem os transtornos alimentares. Essas razões vão desde a insatisfação corporal até a maneira de enfrentar os seus problemas ${ }^{23}$. O presente trabalho pôde mostrar em seus resultados a interferência do relacionamento compulsivo das mães com as filhas. A equipe de saúde relatou que as pacientes do ambulatório descreviam o seu relacionamento com as mães como fusionais e conflituosos caracterizados pela necessidade persistente da presença e companhia frequente da mãe. Esse convívio ia desde o obsessivo, ao indiferente. Um estudo realizado por Leonidas ${ }^{24}$, fala sobre o relacionamento entre mãe e filha a partir de uma dependência emocional mútua, seguindo um padrão de codependência e repleto de conflitos.

Associado a este fato, os resultados do estudo também mostraram o envolvimento das mães em tentativas de burlar o tratamento pelo qual as filhas estavam passando, corroborando a ideia de que recuperar o peso estivesse fazendo mal e causando sofrimento para elas. Em pesquisa realizada com estudantes para analisar o controle alimentar, revelou-se que mesmo com a proibição do comércio de alguns alimentos nos arredores da escola, os alunos fraudavam esta norma e levavam alimentos escondidos em seu material escolar e quando as mães eram questionadas sobre o consumo destes alimentos pelos filhos, elas autorizavam tal conduta para não contraria-los ${ }^{25}$.

Por meio dos relatos, também verificamos que muitas pacientes burlavam o próprio tratamento, relatando que realizam este fato por muitas vezes não conseguir se controlar, tomando atitudes até mesmo inviáveis para se livrar da ingestão alimentar; como por exemplo, o vômito autoinduzido após a alimentação vigiada, esconder a comida dentro da própria roupa, no guarda roupas e dentro do banheiro, o que corrobora outro estudo que mostrou a prática dessas ações ${ }^{24}$. Em um dos casos mais graves relatados na presente pesquisa, a paciente removeu a sua própria sonda alimentar e colocou na janela para dispensar 0 alimento sem que ninguém 0 encontrasse.

Além da automutilação, foi observada nas falas que uma grande parcela das mulheres acometidas por esses transtornos, já tentaram o suicídio. De maneira geral, o fenômeno do suicídio é um grave problema, ocupando um lugar prioritário na pauta dos problemas sociais que provocam impacto sobre a saúde pública do mundo e afeta toda a sociedade.

Por esta razão, o Ministério da Saúde estabeleceu em 2006, a Portaria $1.876^{26}$, na qual: "Institui Diretrizes Nacionais para Prevenção do Suicídio, a ser implantadas em todas as unidades federadas, respeitadas as competências das três esferas de gestão". Mais de 800 mil pessoas tiram a própria vida por ano e no Brasil, esta ocorrência é de 11 mil casos/ano. Devido ao alto índice de lesões autoprovadas no país, a partir do ano de 2011 tornou-se obrigatória a sua notificação. Entre 2011 e 2016 foram notificadas 176.226 mil ocorrências de violência autoinfligida e destas, $27,4 \%$ foram tentativas de suicídio, ocorrendo duas vezes mais entre as mulheres ${ }^{27,28}$.

Desta forma, ressalta-se a grande importância do tema pesquisado, devido ao grande aumento de casos nos últimos anos de mulheres com anorexia e bulimia nervosa. A distorção da imagem corporal associada à baixa 
autoestima são os responsáveis pela busca incessante de emagrecimento e do corpo considerado ideal, tornando as práticas de autoflagelo e automutilação cada vez mais frequentes $^{16,29}$. As ações autolesivas vêm adquirindo um caráter endêmico e se convertendo em um problema de saúde pública em muitos países, pois os setores de saúde vêm enfrentando dificuldades nos serviços de urgência, atenção especializada, reabilitação física, psicológica e de assistência social em consequência desses atos ${ }^{30-32}$.

CONCLUSÃO

A violência autoinfligida mostra-se muito presente entre mulheres com anorexia e bulimia nervosa. Fato este verificado por meio dos discursos e das palavras "suicídio, mutilar e burlar" relatados pela equipe de saúde responsável por estes pacientes no ambulatório de psiquiatria. E apesar da dificuldade de avaliar a sua existência, a violência autoinfligida acarreta uma grande sobrecarga ao serviço de saúde pública, pois esses atos são considerados um importante fator de risco para comportamentos autolesivos graves e para a provável consumação de suicídios.

\section{REFERÊNCIAS}

1. Farah MHS, Mate $\mathrm{CH}$. Practices of anorexia and bulimia as an aesthetics of existence. Educ Pesqui. 2015;41(4):883-98.

2. Gomes R, Silva L. Desordens alimentares e perfeccionismo: um estudo com atletas portuguesas. Psicol Rev. 2010;16(3):469-89.

3. American Psychiatry Association. Diagnostic And statistical manual of mental disorders. 5th ed. Washington: American Psychiatry Association; 2013.

4. Organização Mundial da Saúde. CID-10: Classificação Estatística Internacional de Doenças e Problemas Relacionados à Saúde. 10th ed. São Paulo: Universidade de São Paulo; 1997.

5. Antunes KT, Amaral CF, Balbinot CEA. Anorexia e bulimia nervosa: complicações bucais e o papel do cirurgião-dentista frente a transtornos alimentares. Disc. Scientia. 2007;8(1):159-67.

6. Veloso C, Monteiro CFS, Veloso LUP, Figueiredo MLF, Fonseca RSB, Araújo TME, et al. Violência autoinfligida por intoxicação exógena em um serviço de urgência e emergência. Rev Gaúcha Enferm. 2017;38(2):e66187.

7. Le Breton D. Escarificações na adolescência: uma abordagem antropológica. Horiz Antropol. 2010;16(33):25-40.

8. Magalhães APN, Alves VM, Comassetto I, Lima PC, Faro ACM, Nardi AE. Atendimento a tentativas de suicídio por serviço de atenção pré-hospitalar. J Bras Psiquiatr. 2014;63(1):16-22.

9. Lervolino SA, Pelicioni MCF. A utilização do grupo focal como metodologia qualitativa na promoção da saúde. Rev Esc Enferm USP. 2001;35(2):115-21.

10. Ratinaud P. IRAMUTEQ: Interface de R pour lês analyses multidimensionnelles de textes et de questionnaires [computer software] 2009. [cited 2018 maio 15] Available from: www.iramuteq.org

11. Moura LKB, Marcaccini AM, Matos FTC, Sousa AFL, Nascimento GC, Moura MEB. Revisão Integrativa sobre o câncer bucal. R Pesq Cuid Fundam. 2014;6(5):164-75.

12. Marchand P, Ratinaud P. (2012). L'analyse de similitude appliquée aux corpus textuels: les primaires socialistes pour l'élection présidentielle française (septembre-octobre 2011). Actes des 11eme Journées internationales d'Analyse statistique des Données Textuelles. JADT. 2012:687-699.

13. Kessler RC, Berglund PA, Chiu WT, Deitz AC, Hudson JI, Shahly V, et al. The prevalence and correlates of binge eating disorder in the World Health Organization World Mental Health Surveys. Biol Psychiatry. 2013;73(9):904-14.

14. Coras PM, Araújo APS. O papel da enfermagem no tratamento dos transtornos alimentares do tipo anorexia e bulimia nervosas. UNOPAR Cient Ciênc Biol Saúde. 2011;13:315-24.

15.Lima FPD, Blank VLG, Menegon FA. Prevalence of mental and behavioral disorders in military police of Santa Catarina. Psicol Ciênc Prof. 2015;35(3):824-40.

16. Silva AC, Botti NCL. Comportamento autolesivo ao longo do ciclo vital: Revisão integrativa da literatura. Rev Portug Enfermag Saúde Mental. 2017;(18):67-76.

17. Ross S, Heath NL, Toste JR. Non-suicidal selfinjury and eating pathology in high school students. Am J Orthop. 2009;79(1):83-92.

18. Cucchi A, Ryan D, Konstantakopoulos G, Stroumpa S, Kaçar AŞ, Renshaw S, et al. Lifetime prevalence of non-suicidal self-injury in patients with eating disorders: a systematic review and meta-analysis. Psychol Med. 2016; 46(7):1345-58.

19.Venosa VS. $O$ ato de cortar-se: uma investigação psicanalítica a partir do caso Amanda e do caso Catarina [dissertação]. São Paulo: Universidade de São Paulo; 2015.

20. Pinaud AB, Seguy C, Thibaut F. Self-mutilating behaviour: a study on 30 inpatients. Encephale. 2009;35(6):538-43.

21. Pommereau $X$. Les violences cutanées autoinfligées à l'adolescence. Enfances Psy. 2006;32(3):58-71. 
22. Garel P. L'automutilationsuperficielle à l'adolescence: le corps dans tous ses états. Cahiers Critiques de Thérapie Familiale et de Pratiques de Réseaux. 2008;40(1):227-35.

23. Alves E, Vasconcelos FDAGD, Calvo MCM, Neves JD. Prevalência de sintomas de anorexia nervosa e insatisfação com a imagem corporal em adolescentes do sexo feminino do Município de Florianópolis, Santa Catarina, Brasil. Cad Saúde Pública. 2008;24(3):503-12.

24. Leonidas C, Santos MA. Relações familiares nos transtornos alimentares: o Genograma como instrumento de investigação. Ciênc Saúde Coletiva. 2015;20(5):1435-47.

25. Dutra RCDA. Consumo alimentar infantil: quando a criança é convertida em sujeito. Soc Estado. 2015;30(2):451-69.

26. Ministério da Saúde (BR). Portaria no 1.876, de 14 de agosto de 2006. Institui Diretrizes Nacionais para Prevenção do Suicídio, a ser implantadas em todas as unidades federadas, respeitadas as competências das três esferas de gestão. Brasília (DF); 2006 [cited 2018 Jan 23]. Available from: http://bvsms.saude.gov.br/bvs/saudelegis/gm/2 006/prt1876_14_08_2006.html

27. World Health Organization. Preventing suicide: a global imperative. Geneva: WHO; 2014.

28. Ministério da Saúde (BR). Secretaria de Vigilância em Saúde. Suicídio: saber, agir e prevenir. Brasília: Ministério da Saúde; 2017.

29. Tossati AM, Peres L, Preissiler H. Imagem corporal e as influências para os transtornos alimentares nas adolescentes jovens. Rev Bras Obes Nutr Emagrec. 2007;1(4):34-47.

30. Organización Panamericana de la Salud. Las condiciones de salud en las Américas. Washington Organización Panamericana de la Salud; 1994.

31. Mendonça MFS, Ludermir AB. Violência por parceiro íntimo e incidência de transtorno mental comum. Rev Saúde Pública. 2017;51(32):1-8.

32. Leite FMC, Amorim MHC, Wehrmeister FC, Gigante DP. Violência contra a mulher em Vitória, Espírito Santo, Brasil. Rev Saúde Pública. 2017;51(33):1-12.

\section{CONFLITO DE INTERESSES}

Os autores declaram não haver conflitos de interesse

\section{AUTOR PARA CORRESPONDÊNCIA}

\author{
Ronald Jefferson Martins \\ NEPESCO - Núcleo de Pesquisa em Saúde Coletiva \\ Faculdade de Odontologia de Araçatuba \\ UNESP - Univ Estadual Paulista \\ Departamento de Odontologia Preventiva e Restauradora \\ R. José Bonifácio, nํ 1193 - Vila Mendonça \\ 16015-050 - Araçatuba-SP - Brasil \\ Tel. (55 018) 3636-2824 \\ E-mail: ronald.j.martins@unesp.br
}

Submetido em 09/09/2021 Aceito em 04/11/2021 